\title{
Abundant phase transitions
}

\section{The bewildering variety in which phase transitions come is neatly illustrated in a collection of papers to be found in a single issue of a journal.}

LAST year's final issue (on 26 December 1983) of Physical Review Letters, a journal which is no longer the exclusive preserve of high-energy physicists, contains half a dozen new twists in the understanding of phase transitions. What follows is merely a selection.

Simplest, perhaps because it is experimental, is a study of the properties of plutonium antimonide (chemical formula PuSb) which, among other things, has entailed the preparation of what are said to be the first "sizeable crystals" of any plutonium compounds - they come out at 8 $\mathrm{mm}^{3}$. That in itself has been a substantial undertaking. Crystals of PuSb have been grown and embedded in a matrix of metallic copper (B.R. Cooper et al. Phys. Rev. Lett. 51,2418 ) by a four-cornered collaboration involving the Los Alamos National Laboratory, the University of West Virginia, ETH at Zurich and the Transuranium Institute at Karlsruhe (on account of its "unique handling and glove-box facilities"). The trick of surrounding the crystals with solid copper has the advantages of eliminating the toxicity hazard of the plutonium but also of preventing self-heating due to spontaneous fission.

The interest of PuSb is its magnetism. Plutonium is an element whose $5 f$ shell is only partly filled with electrons. Indeed, the triply charged plutonium ions in the antimonide can have only five $5 f$ electrons, whence the argument that the ion must have much in common with the electronic structure of the trivalent cerium ion $\mathrm{Ce}^{3+}$, which has only one electron in the $4 f$ shell.

This expectation seems to be borne out by experiment. PuSb, a cubic crystal, has markedly anisotropic properties which imply that the $f$ electrons are not strictly localized on the atoms which contribute them to the lattice but, rather, are stretched out towards their nearest neighbours. Cooper et al. argue that this is an interesting case of hybridization between atomic and band electrons. Their discussion of the strength of the magnetic coupling between neighbouring plutonium atoms in the lattice is necessarily incomplete, but does not obscure the striking circumstance that $\mathrm{PuSb}$ is a ferromagnetic material below $75 \mathrm{~K}$ and an antiferromagnetic material above, with a transition to disorder at $85 \mathrm{~K}$.

The obvious interest of the data now published is that they offer a way of testing different methods of approximation to interionic forces - and that there are more where these have come from. The antiferromagnetic phase of PuSb appears from neutron diffraction to have long-range order in which magnetic spins cancel out over a distance of about two lattice units.

This is comparable with the nature of the long-range order with respect to translation that occurs in the nematic phase of liquid crystals, where planar (and usually polar) molecules are spatially ordered. In the same issue of Phys. Rev. Lett. (p.2386), G. Grinstein and John Toner from the IBM Research Center at Yorktown Heights, New York, claim a remarkable prediction of a previously unrecognized property of those liquid crystals which can exist in the nematic phase and the two smectic phases $a$ and $c$, the second of which involves both orientational and translation ordering of the planar molecules.

Which of the three phases will be assumed by any material will be a function of the only two adjustable parameters available - the temperature and the concentration of the material in its solvent. On such a plot, the pairwise phase boundaries should come together at a triple point, but so far this occurrence seems to have been unexplored. Grinstein and Toner argue that there cannot be a simple triple point but, rather, that there must be a fourth phase, a kind of hybrid between the nematic and smectic $c$.

Physically, what this implies is that the geometrical rearrangement of the planar molecules needed to accomplish the phase transition requires more than two steps to be taken in succession, but the point is established by means of A. C. Wilson's correspondence between lattice statistics and the renormalization theories that usually turn up in gauge field theories. None of this, of course, implies that the fourth phase will exist over a wide range of concentration or of temperature indeed, the predicted effect may turn out to be too fleeting to be recognized.

The solidification of xenon adsorbed in gaseous form on a platinum surface has, however, been neatly observed by the scattering of helium atoms. Bene Prelsema, Laurens K. Verheij and George Comsa of the Low-Temperature and Vacuum Physics Division of the West German nuclear research centre at Julich point out (p.2410) that the cross-section for scattering helium atoms from molecules (for example $\mathrm{CO}$ ) adsorbed on a metal surface can be very much larger than simple geometry suggests, perhaps as much as $125 \AA^{2}$. The result is that it is possible to use helium scattering as a measure of aggregation of adsorbed molecules for each possible density of coverage. Hitherto, attempts to measure condensation in adsorbed xenon have been unsuccessful, chiefly because of the need to work with surfaces which have been densely covered with adsorbed molecules. The helium atom technique works, but at the opposite end of the range, with as few as one in a thousand adsorption sites occupied. The result is what must have been hoped for. The transition from twodimensional gas to two-dimensional solid occurs at lower coverage by adsorbed gas with decreasing temperature (at least down to that of liquid nitrogen). It has even been possible to infer the heat of vaporization of solid two-dimensional xenon $(1.1 \mathrm{kcal}$ per mole) and, because the densities of adsorbed gas at which crystallization occurs are indeed low, to explain why the phenomenon has not been previously observed. While xenon itself is of moderate interest, the helium scattering technique is likely to be widely used.

No fewer than three other types of phase transitions crop up in the same issue of Phys. Rev. Lett., of which the transition known as commensurate-incommensurate is perhaps the simplest. A. H. Moudden, D. E. Moncton and J. D. Axe from the Brookhaven National Laboratory describe (p.2390) measurements of the behaviour of the dipolar molecular thiourea in strong electric fields using $\mathrm{X}$ rays to look for periodicity in a crystal lattice. At high field strength, greater than 1,000 volts per $\mathrm{mm}$, thiourea crystals behave as if successful slab-like domains in which dipoles are oriented in one direction or the opposite in an alternating fashion along the field. The numbers suggest that under these conditions the slabs may be either 7 or 8 molecular planes thick. Decreasing the electric field allows 9-fold slabs to occur and also suggests how the relaxation to low field conditions may show hysteresis, as is observed.

What else? The process of wetting a solid surface is also a candidate for phase transition while the problem of percolation, in which free moving molecules thread their way through a tortuous lattice, clogging the system if there are too many of them, is dealt with for the first time on a lattice of fractal dimensions, no doubt simulating more accurately what may happen in the percolation of petroleum through, say, a reservoir rock.

John Maddox 